\title{
New Calculation of Secondary Antiprotons in Cosmic Rays
}

\author{
I. V. Moskalenko ${ }^{* a}$ G. Jóhannesson, ${ }^{b}$ M. Kachelrieß,,${ }^{c}$ E. Orlando, ${ }^{a}$ \\ S. S. Ostapchenko, ${ }^{d, e}$ T. A. Porter ${ }^{a}$
}

${ }^{a}$ Hansen Experimental Physics Laboratory and Kavli Institute for Particle Astrophysics and Cosmology, Stanford University, Stanford, CA 94305, U.S.A.

${ }^{b}$ Science Institute, University of Iceland, Dunhaga 5, IS-107 Reykjavík, Iceland

${ }^{c}$ Institutt for fysikk, NTNU, 7491 Trondheim, Norway

${ }^{d}$ Frankfurt Institute for Advanced Studies, 60438 Frankfurt am Main, Germany

e Skobeltsyn Institute of Nuclear Physics, Moscow State University, 119991 Moscow, Russia

E-mail: imos@stanford.edu, gudlaugueglast2. stanford.edu,

mika@tf.phys.ntnu.no, eorlandoestanford.edu,

sergeidtf.phys.ntnu.no, tporterestanford.edu

A dramatic increase in the accuracy and statistics of space-borne cosmic ray (CR) measurements has yielded several breakthroughs over the last several years. The most puzzling is the rise in the positron fraction above $10 \mathrm{GeV}$ over the predictions of the propagation models assuming pure secondary production. Antiprotons are produced in CR interactions with interstellar gas and are, therefore, called secondary. These are the same interactions that produce charged and neutral mesons which decay to secondary electrons and positrons and $\gamma$-rays. However, in contrast to CR electrons and positrons that can be produced copiously in pulsars, there is no known astrophysical source of primary $\bar{p}$. Therefore, $\bar{p}$ data and their correct interpretation hold the key to the resolution of many astrophysical puzzles. We calculated $\bar{p}$ production in $p p^{-}, p A-$, and $A A$-interactions using EPOS-LHC and QGSJET-II-04, two of the most advanced Monte Carlo generators tuned to accelerator data including those from the LHC. The new cross sections are being incorporated into the GALPROP code to allow an accurate calculation of the spectrum of secondary $\bar{p}$ in CRs.

The 34th International Cosmic Ray Conference,

30 July- 6 August, 2015

The Hague, The Netherlands

\footnotetext{
* Speaker.
} 


\section{Introduction}

Antiprotons in CRs are produced in CR interactions with interstellar gas and are, therefore, called secondary. The same interactions produce charged and neutral mesons that decay to secondary $e^{ \pm}$and $\gamma$-rays. However, in contrast to $\mathrm{CR}$ positrons that can be produced copiously in pulsars, there is no known astrophysical source of primary $\bar{p}$. Since the $\bar{p}$ "background" is fairly low this provides an opportunity to search for a new phenomenon or an exotic signal if the CR $\bar{p}$ flux is measured accurately.

Recent discoveries in astrophysics of CRs, however, have changed the landscape dramatically. The most important was a clear confirmation of the rise in the positron fraction by PAMELA [1,2] - relative to expectations as if all positrons in CRs are secondary [3, 4] - that was earlier noticed by the HEAT collaboration [5]. This was subsequently confirmed and extended to higher energies by Fermi-LAT [6], and measured with even greater precision and extended to even higher energies by AMS-02 [7]. Most recently, the measurement of the positron fraction has been extended up to $500 \mathrm{GeV}$ by the AMS- 02 collaboration hinting for some flattening of the fraction above $200 \mathrm{GeV}$ [8], but the statistics is still low in this energy range. New accurate $\bar{p}$ measurements were done by PAMELA $[9,10]$ covering the range from $70 \mathrm{MeV}-200 \mathrm{GeV}$, and more is expected from AMS02. Above a few $\mathrm{GeV}$, the data are consistent with secondary production, in strong contrast with positron results. Another important milestone is an accurate measurement of the $\mathrm{B} / \mathrm{C}$ ratio up to $100 \mathrm{GeV} /$ nucleon by PAMELA [11] that is consistent with preliminary AMS- $02^{1}$ measurements reaching $\sim 400 \mathrm{GeV} /$ nucleon. Both measurements indicate the index of the diffusion coefficient $\alpha \approx 0.4$ or even smaller [11] that supports a Kolmogorov-type power spectrum of interstellar turbulence [12], thus favoring the stochastic reacceleration model for interstellar propagation. Besides providing more accurate data over a wider energy range these new measurements give an important insight into CR acceleration and propagation processes. Also relevant are the new measurements of CR proton and He spectra, the most abundant CR species. Combined measurements by PAMELA [13], ATIC [14], and CREAM [15] hint on a break in both spectra at about the same rigidity $\sim 230 \mathrm{GV}$. The flattening of the $\mathrm{CR}$ proton spectrum at high energies was also confirmed by the Fermi-LAT through observations of $\gamma$-ray emission of the Earth's limb [16]. Recently, precise measurements of the proton spectrum up to $\sim 2 \mathrm{TeV}$ were reported by the AMS-02 team [17].

Antiproton data and their correct interpretation hold the key to the resolution of many astrophysical puzzles. If the rise in the positron fraction is due to WIMP annihilations, $\bar{p}$ data provide important constraints on WIMP models [18, 19], for a review, see [20]. If the rise is due to conventional astrophysics, $\bar{p}$ and $\mathrm{B} / \mathrm{C}$ measurements extended to higher energies may be able to discriminate between the pulsar [21]-[25] and SNR hypotheses [26, 27]. The latter proposes a secondary component with a hard energy spectrum that is produced in a SNR shock by accelerated protons. It also predicts a rise [26]-[30] or flattening [31, 32] in all secondaries, such as the $\bar{p} / p$ and $\mathrm{B} / \mathrm{C}$ ratios, at high energies.

The accuracy of the $\bar{p}$ production cross section is critical for astrophysical applications and searches for new physics. This is especially true in view of many expectations connected with the upcoming data releases by the AMS-02 experiment operating at the International Space Station, and by soon-to-be-launched ISS-CREAM, CALET and GAPS experiments. This holds even more for

\footnotetext{
${ }^{1}$ http://www.ams02.org/2013/07/new-results-from-ams-presented-at-icrc-2013/
} 

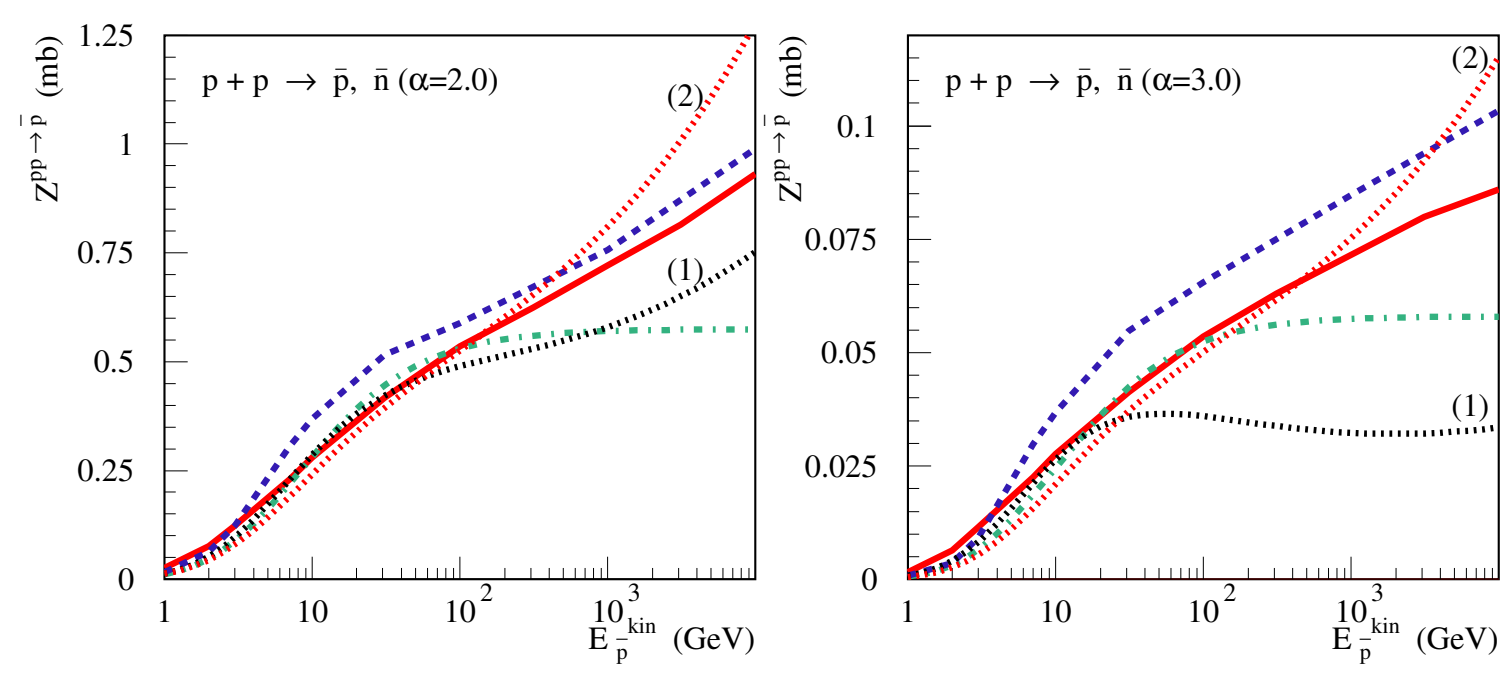

Figure 1: Energy dependence of $Z$-factors for $\bar{p}$ and $\bar{n}$ production, $Z_{\bar{p}}^{p p}\left(E_{\bar{p}}, \alpha\right)$ (plotted as a function of $\left.E_{\bar{p}}^{\mathrm{kin}}\right)$, for $\alpha=2$ (left) and $\alpha=3$ (right), as calculated with QGSJET-IIm (solid, red) and EPOS-LHC (dashed, blue), or using the parameterizations by [36] (dot-dashed, green) and [37] (dotted; the lines marked "(1)" (black) and "(2)" (red) correspond to the respective parameter sets).

new opportunities that would open up with antideuteron detection in CRs. In turn, the calculation of antideuteron production relies on the inclusive $\bar{p}$ production cross sections and the detailed knowledge of two-particle correlations [33, 34].

In this work, we analyzed $\bar{p}$ production in $p p-, p A-$, and $A A$-interactions using EPOS-LHC and QGSJET-II-04, two of the most advanced Monte Carlo (MC) generators tuned to numerous accelerator data including those from the Large Hadron Collider (LHC). The $\bar{p}$ yields obtained with these MC generators differ by a factor of few from yields of parameterizations commonly used in astrophysics. More details can be found in [35].

\section{2. $Z$-factors for $\bar{p}$ production}

To compare the impact of different interaction models and parameterizations on the predicted CR $\bar{p}$ spectrum, it is convenient, similarly to the $\gamma$-ray case [38], to use the corresponding " $Z$ factors." They are defined as the spectrally averaged energy fraction transferred to $\bar{p}$ in $p p^{-}, p A^{-}$, $A p$-, or $A A$-collisions, assuming that the spectra of CR species in the relevant energy range can be approximated by a power-law, $I_{i}(E)=K_{i} E^{-\alpha_{i}}$. Then the contribution $q_{\bar{p}}^{i j}\left(E_{\bar{p}}\right)$ to the flux of secondary CRs (here, $\bar{p}$ ) from interactions of the CR species $i$ with ISM component $j(i, j=$ protons or nuclei) of number density $n_{j}$,

$$
q_{\bar{p}}^{i j}\left(E_{\bar{p}}\right)=n_{j} \int_{E_{\mathrm{thr}}\left(E_{\bar{p}}\right)}^{\infty} d E \frac{d \sigma^{i j \rightarrow \bar{p}}\left(E, E_{\bar{p}}\right)}{d E_{\bar{p}}} I_{i}(E),
$$

can be rewritten as [38]

$$
q_{\bar{p}}^{i j}\left(E_{\bar{p}}\right)=n_{j} I_{i}\left(E_{\bar{p}}\right) Z_{\bar{p}}^{i j}\left(E_{\bar{p}}, \alpha_{i}\right)
$$




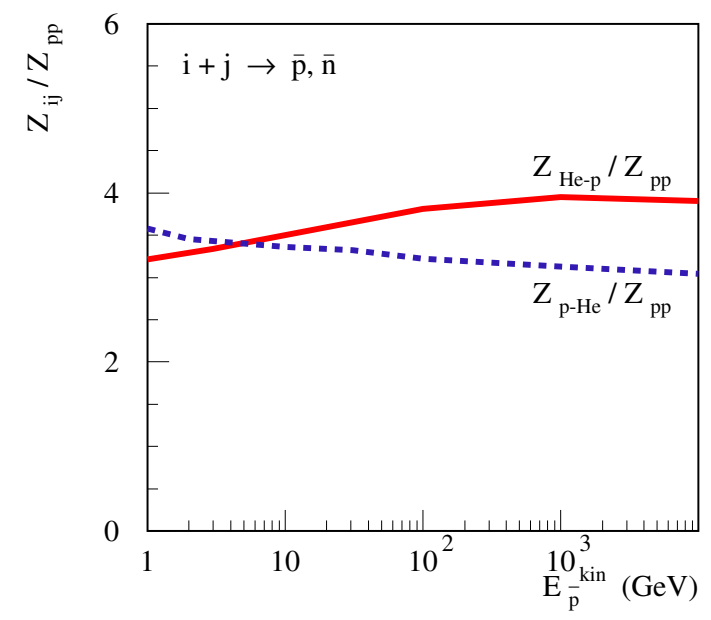

Figure 2: Energy dependence of the enhancement of the He $p$ (solid, red) and $p \mathrm{He}$ (dashed, blue) contributions to the $\bar{p}$ spectrum, relative to the $p p$-case, $Z_{\bar{p}}^{i j}\left(E_{\bar{p}}, \alpha\right) / Z_{\bar{p}}^{p p}\left(E_{\bar{p}}, \alpha\right)$ (plotted as a function of $\left.E_{\bar{p}}^{\mathrm{kin}}\right)$, for $\alpha=2.6$.

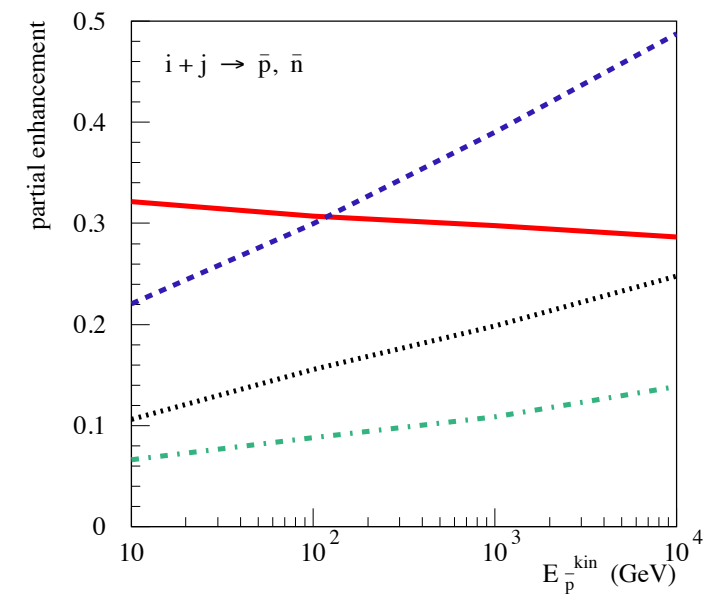

Figure 3: Energy dependence of partial contributions $\varepsilon_{i j}^{\bar{p}}\left(E_{\bar{p}}\right)$ to the nuclear enhancement factor from different interaction channels: $p \mathrm{He}$ (solid, red), He $p$ (dashed, blue), He He (dot-dashed, green), and all others (dotted, black); the CR composition given in Table 2 is used.

Here, we expressed the $Z$-factor $Z_{\bar{p}}^{i j}$ via the inclusive spectra of antiprotons $d \sigma^{i j \rightarrow \bar{p}}\left(E, z_{\bar{p}}\right) / d z_{\bar{p}}$, $z_{\bar{p}}=E_{\bar{p}} / E$, as

$$
Z_{\bar{p}}^{i j}\left(E_{\bar{p}}, \alpha\right)=\int_{0}^{1} d z z^{\alpha-1} \frac{d \sigma^{i j \rightarrow \bar{p}}\left(E_{\bar{p}} / z, z\right)}{d z},
$$

where $E$ corresponds to the energy per nucleon for nuclear projectiles. The $Z$-factors $Z_{\bar{p}}^{i j}$ depend both on the $\bar{p}$ production spectra and on the spectral slopes $\alpha_{i}$, containing all the dependences on hadronic interaction models. However, these factors are independent of the $\mathrm{CR}$ abundances.

As follows from Eq. (2.2) and the more detailed discussion by [38], the respective partial enhancements compared to the yield from $p p$-interactions are proportional to the corresponding Z-factors,

$$
\varepsilon_{i j}^{\bar{p}}\left(E_{\bar{p}}\right)=\frac{q_{\bar{p}}^{i j}\left(E_{\bar{p}}\right)}{q_{\bar{p}}^{p p}\left(E_{\bar{p}}\right)}=\frac{n_{j}}{n_{p}} \frac{I_{i}\left(E_{\bar{p}}\right)}{I_{p}\left(E_{\bar{p}}\right)} \frac{Z_{\bar{p}}^{i j}\left(E_{\bar{p}}, \alpha_{i}\right)}{Z_{\bar{p}}^{p} p\left(E_{\bar{p}}, \alpha_{p}\right)} .
$$

In particular, contributions of CR nuclei may be additionally enhanced, if the spectral indices $\alpha_{i}<$ $\alpha_{p}$, because of the strong $\alpha$-dependence of the $Z$-factors [38].

The calculated energy dependence of the $Z$-factors for $\bar{p}$ production $^{2}, Z_{\bar{p}}^{p p}\left(E_{\bar{p}}, \alpha\right)$, is compared in Fig. 1 for QGSJET-IIm, EPOS-LHC, and the parameterizations from [36] and [37]. Two values, $\alpha=2$ and $\alpha=3$, for the slope of the CR proton spectrum are considered, which bracket the physically most interesting range.

The energy dependence of the Z-factors is affected by threshold effects (Fig. 2). As a result, the partial contributions of CR nuclei in the region of relatively small $E_{\bar{p}}$ are suppressed. This suppression diminishes at higher energies, where one approaches the asymptotic limit.

\footnotetext{
${ }^{2}$ Here we take into account both $\bar{p}$ and $\bar{n}$ production when calculating $Z$-factors; for brevity, we use the same notation $Z_{\bar{p}}^{p p}$ instead of $Z_{\bar{p}+\bar{n}}^{p p}$. For the parameterizations by [36] and [37], we double the $\bar{p}$ yields.
} 


\begin{tabular}{|c|c|c|c|c|c|c|c|c|}
\hline \multirow[b]{2}{*}{$E_{\bar{p}}^{\mathrm{kin}}, \mathrm{GeV}$} & \multirow[b]{2}{*}{ Projectile } & \multirow[b]{2}{*}{ Target } & \multicolumn{6}{|c|}{$Z_{\bar{p}}^{i j}\left(E_{\bar{p}}, \alpha\right), \mathrm{mb}$} \\
\hline & & & $\alpha=2$ & $\alpha=2.2$ & $\alpha=2.4$ & $\alpha=2.6$ & $\alpha=2.8$ & $\alpha=3$ \\
\hline 1 & $p$ & $p$ & 0.0254 & 0.0138 & 0.00772 & 0.00441 & 0.00258 & 0.00153 \\
\hline 1 & $\mathrm{He}$ & $p$ & 0.0808 & 0.0442 & 0.0248 & 0.0143 & 0.00838 & 0.00501 \\
\hline 1 & $\mathrm{CNO}(A=14)$ & $p$ & 0.184 & 0.101 & 0.0567 & 0.0326 & 0.0192 & 0.0115 \\
\hline 1 & $\mathrm{Mg}-\mathrm{Si}(A=25)$ & $p$ & 0.273 & 0.150 & 0.0845 & 0.0486 & 0.0286 & 0.0171 \\
\hline 1 & $\mathrm{Fe}(A=56)$ & $p$ & 0.447 & 0.245 & 0.138 & 0.0792 & 0.0465 & 0.0278 \\
\hline 1 & $p$ & $\mathrm{He}$ & 0.0919 & 0.0498 & 0.0277 & 0.0158 & 0.00920 & 0.00546 \\
\hline 1 & $\mathrm{He}$ & $\mathrm{He}$ & 0.271 & 0.147 & 0.0824 & 0.0472 & 0.0276 & 0.0165 \\
\hline 1 & $\mathrm{CNO}(A=14)$ & $\mathrm{He}$ & 0.649 & 0.352 & 0.196 & 0.112 & 0.0654 & 0.0389 \\
\hline 1 & $\operatorname{Mg}-\mathrm{Si}(A=25)$ & $\mathrm{He}$ & 0.933 & 0.506 & 0.282 & 0.161 & 0.0937 & 0.0556 \\
\hline 1 & $\mathrm{Fe}(A=56)$ & $\mathrm{He}$ & 1.53 & 0.834 & 0.468 & 0.269 & 0.158 & 0.0944 \\
\hline 10 & $p$ & $p$ & 0.279 & 0.164 & 0.100 & 0.0633 & 0.0413 & 0.0276 \\
\hline 10 & $\mathrm{He}$ & $p$ & 0.979 & 0.573 & 0.350 & 0.222 & 0.144 & 0.0964 \\
\hline 10 & $\mathrm{CNO}(A=14)$ & $p$ & 2.67 & 1.58 & 0.978 & 0.624 & 0.410 & 0.276 \\
\hline 10 & $\mathrm{Mg}-\mathrm{Si}(A=25)$ & $p$ & 4.22 & 2.50 & 1.54 & 0.977 & 0.639 & 0.428 \\
\hline 10 & $\mathrm{Fe}(A=56)$ & $p$ & 7.78 & 4.63 & 2.87 & 1.84 & 1.21 & 0.815 \\
\hline 10 & $p$ & $\mathrm{He}$ & 0.970 & 0.560 & 0.339 & 0.213 & 0.138 & 0.0917 \\
\hline 10 & $\mathrm{He}$ & $\mathrm{He}$ & 3.16 & 1.83 & 1.11 & 0.695 & 0.449 & 0.298 \\
\hline 10 & $\mathrm{CNO}(A=14)$ & $\mathrm{He}$ & 9.16 & 5.33 & 3.24 & 2.04 & 1.32 & 0.875 \\
\hline 10 & $\mathrm{Mg}-\mathrm{Si}(A=25)$ & $\mathrm{He}$ & 14.5 & 8.45 & 5.16 & 3.26 & 2.12 & 1.41 \\
\hline 10 & $\mathrm{Fe}(A=56)$ & $\mathrm{He}$ & 26.0 & 15.2 & 9.31 & 5.90 & 3.85 & 2.57 \\
\hline 100 & $p$ & $p$ & 0.535 & 0.308 & 0.187 & 0.119 & 0.0789 & 0.0536 \\
\hline 100 & $\mathrm{He}$ & $p$ & 2.03 & 1.17 & 0.715 & 0.455 & 0.300 & 0.204 \\
\hline 100 & $\mathrm{CNO}(A=14)$ & $p$ & 6.21 & 3.61 & 2.20 & 1.40 & 0.926 & 0.628 \\
\hline 100 & $\mathrm{Mg}-\mathrm{Si}(A=25)$ & $p$ & 10.6 & 6.19 & 3.79 & 2.42 & 1.60 & 1.09 \\
\hline 100 & $\mathrm{Fe}(A=56)$ & $p$ & 21.8 & 12.7 & 7.80 & 4.99 & 3.30 & 2.25 \\
\hline 100 & $p$ & $\mathrm{He}$ & 1.79 & 1.02 & 0.612 & 0.385 & 0.251 & 0.169 \\
\hline 100 & $\mathrm{He}$ & $\mathrm{He}$ & 6.41 & 3.66 & 2.21 & 1.39 & 0.914 & 0.619 \\
\hline 100 & $\mathrm{CNO}(A=14)$ & $\mathrm{He}$ & 20.8 & 11.9 & 7.15 & 4.50 & 2.94 & 1.98 \\
\hline 100 & $\mathrm{Mg}-\mathrm{Si}(A=25)$ & $\mathrm{He}$ & 35.2 & 20.2 & 12.2 & 7.69 & 5.04 & 3.40 \\
\hline 100 & $\mathrm{Fe}(A=56)$ & $\mathrm{He}$ & 72.0 & 41.3 & 24.9 & 15.7 & 10.2 & 6.87 \\
\hline 1000 & $p$ & $p$ & 0.721 & 0.410 & 0.248 & 0.158 & 0.105 & 0.0715 \\
\hline 1000 & $\mathrm{He}$ & $p$ & 2.79 & 1.60 & 0.978 & 0.625 & 0.416 & 0.286 \\
\hline 1000 & $\mathrm{CNO}(A=14)$ & $p$ & 8.87 & 5.12 & 3.12 & 1.99 & 1.32 & 0.903 \\
\hline 1000 & $\operatorname{Mg}-\mathrm{Si}(A=25)$ & $p$ & 15.3 & 8.81 & 5.37 & 3.42 & 2.27 & 1.55 \\
\hline 1000 & $\mathrm{Fe}(A=56)$ & $p$ & 31.4 & 18.1 & 11.1 & 7.09 & 4.72 & 3.24 \\
\hline 1000 & $p$ & $\mathrm{He}$ & 2.35 & 1.32 & 0.787 & 0.495 & 0.324 & 0.221 \\
\hline 1000 & $\mathrm{He}$ & $\mathrm{He}$ & 8.62 & 4.85 & 2.90 & 1.82 & 1.19 & 0.805 \\
\hline 1000 & $\mathrm{CNO}(A=14)$ & $\mathrm{He}$ & 29.0 & 16.4 & 9.81 & 6.18 & 4.05 & 2.75 \\
\hline 1000 & $\mathrm{Mg}-\mathrm{Si}(A=25)$ & $\mathrm{He}$ & 48.1 & 27.0 & 16.0 & 10.0 & 6.50 & 4.36 \\
\hline 1000 & $\mathrm{Fe}(A=56)$ & $\mathrm{He}$ & 103 & 58.1 & 34.9 & 22.1 & 14.5 & 9.85 \\
\hline 10000 & $p$ & $p$ & 0.931 & 0.516 & 0.307 & 0.193 & 0.127 & 0.0860 \\
\hline 10000 & $\mathrm{He}$ & $p$ & 3.60 & 2.02 & 1.20 & 0.754 & 0.493 & 0.334 \\
\hline 10000 & $\mathrm{CNO}(A=14)$ & $p$ & 11.5 & 6.46 & 3.87 & 2.44 & 1.61 & 1.09 \\
\hline 10000 & $\operatorname{Mg}-\mathrm{Si}(A=25)$ & $p$ & 19.9 & 11.1 & 6.61 & 4.12 & 2.68 & 1.80 \\
\hline 10000 & $\mathrm{Fe}(A=56)$ & $p$ & 41.6 & 23.4 & 13.9 & 8.72 & 5.69 & 3.84 \\
\hline 10000 & $p$ & $\mathrm{He}$ & 3.02 & 1.65 & 0.959 & 0.587 & 0.376 & 0.249 \\
\hline 10000 & $\mathrm{He}$ & $\mathrm{He}$ & 11.1 & 6.14 & 3.61 & 2.24 & 1.46 & 0.983 \\
\hline 10000 & $\mathrm{CNO}(A=14)$ & $\mathrm{He}$ & 37.4 & 20.5 & 12.0 & 7.40 & 4.77 & 3.20 \\
\hline 10000 & $\mathrm{Mg}-\mathrm{Si}(A=25)$ & $\mathrm{He}$ & 63.7 & 35.0 & 20.5 & 12.7 & 8.18 & 5.48 \\
\hline 10000 & $\mathrm{Fe}(A=56)$ & $\mathrm{He}$ & 135 & 74.5 & 43.6 & 27.0 & 17.4 & 11.6 \\
\hline
\end{tabular}

Table 1: $Z$-factors for $(\bar{p}+\bar{n})$ production, $Z_{\bar{p}}^{i j}\left(E_{\bar{p}}, \alpha\right)$, calculated with QGSJET-IIm. 


\begin{tabular}{cccccc}
\hline & \multicolumn{5}{c}{ Groups of nuclei } \\
\cline { 2 - 6 } Parameters & $\mathrm{H}(A=1)$ & $\mathrm{He}(A=4)$ & $\mathrm{CNO}(A=14)$ & $\mathrm{Mg}-\mathrm{Si}(A=25)$ & $\mathrm{Fe}(A=56)$ \\
$K$ & 14900 & 600 & 33.2 & 34.2 & 4.45 \\
$\alpha$ & 2.74 & 2.64 & 2.60 & 2.79 & 2.68 \\
\hline
\end{tabular}

Table 2: Spectral parameterizations for groups of CR nuclei [39].

Table 1 shows the calculated $Z$-factors $Z_{\bar{p}}^{i j}$ for different $E_{\bar{p}}^{\mathrm{kin}}$, slopes $\alpha$, and different combinations of CR and ISM nuclei. These results may be used for the calculation of secondary $\bar{p}$ spectra when the partial spectra of CR mass groups can be approximated by a power law behavior in the corresponding energy range.

The partial contributions $\varepsilon_{i j}^{\bar{p}}\left(E_{\bar{p}}\right)$ to the nuclear enhancement factor from different interaction channels are shown in Fig. 3.

\section{GALPROP code}

The new $\bar{p}$ production cross sections are being incorporated into the GALPROP code to allow an accurate calculation of the spectrum of secondary $\bar{p}$ in CRs.

The GALPROP model has established itself as a standard self-consistent model for CR propagation in the Galaxy and associated diffuse emissions (radio, microwave, X-rays, $\gamma$-rays) that is widely used by the astrophysical community - the experimental teams and individuals. The key concept underlying the GALPROP code is that various kinds of data, e.g., direct CR measurements including primary and secondary nuclei, electrons and positrons, $\gamma$-rays, synchrotron radiation, and so forth, are all related to the same astrophysical components of the Galaxy and hence have to be modeled self-consistently [40]. The goal is for GALPROP-based models to be as realistic as possible and to make use of available astronomical information, nuclear and particle data, with a minimum of simplifying assumptions. A full description of the rationale and motivation is given in the review [41]. The GALPROP code is available from a dedicated website ${ }^{3}$ where a $500+$ core facility for users to run the code via online forms in a web-browser (WebRun) is also provided [42].

\section{Acknowledgments}

This work is supported through NASA Grants No. NNX13AO92G, and NNX13AC47G.

\section{References}

[1] O. Adriani et al., An anomalous positron abundance in cosmic rays with energies 1.5-100 GeV, Nature 458 (2009) 607-609, [arXiv: 0810 . 4995].

[2] O. Adriani et al., Cosmic-Ray Positron Energy Spectrum Measured by PAMELA, PRL 111 (2013) 081102, [arXiv:1308.0133].

[3] R. J. Protheroe, On the nature of the cosmic ray positron spectrum, ApJ 254 (1982) 391-397.

\footnotetext{
${ }^{3}$ http://galprop.stanford.edu
} 
[4] I. V. Moskalenko and A. W. Strong, Production and Propagation of Cosmic-Ray Positrons and Electrons, ApJ 493 (1998) 694-707, [astro-ph/ 9710124 ].

[5] J. J. Beatty et al., New Measurement of the Cosmic-Ray Positron Fraction from 5 to 15 GeV, PRL 93 (2004) 241102, [astro-ph/0412230].

[6] M. Ackermann et al., Measurement of Separate Cosmic-Ray Electron and Positron Spectra with the Fermi Large Area Telescope, PRL 108 (2012) 011103, [arXiv: 1109.0521 ].

[7] M. Aguilar and et al., First Result from the Alpha Magnetic Spectrometer on the International Space Station: Precision Measurement of the Positron Fraction in Primary Cosmic Rays of 0.5-350 GeV, PRL 110 (2013) 141102.

[8] M. Aguilar and et al., Precision Measurement of the $\left(e^{+}+e^{-}\right)$Flux in Primary Cosmic Rays from 0.5 GeV to $1 \mathrm{TeV}$ with the Alpha Magnetic Spectrometer on the International Space Station, PRL 113 (2014) 221102.

[9] O. Adriani et al., PAMELA Results on the Cosmic-Ray Antiproton Flux from $60 \mathrm{MeV}$ to $180 \mathrm{GeV}$ in Kinetic Energy, PRL 105 (2010) 121101, [arXiv: 1007.0821 ].

[10] O. Adriani et al., Measurement of the flux of primary cosmic ray antiprotons with energies of $60 \mathrm{MeV}$ to $350 \mathrm{GeV}$ in the PAMELA experiment, Soviet JETP Letters 96 (2013) 621-627.

[11] O. Adriani et al., Measurement of Boron and Carbon Fluxes in Cosmic Rays with the PAMELA Experiment, ApJ 791 (2014) 93, [arXiv:1407.1657].

[12] A. Kolmogorov, The Local Structure of Turbulence in Incompressible Viscous Fluid for Very Large Reynolds' Numbers, Akademiia Nauk SSSR Doklady 30 (1941) 301-305.

[13] O. Adriani et al., PAMELA Measurements of Cosmic-Ray Proton and Helium Spectra, Science 332 (2011) 69-, [arXiv:1103.4055].

[14] A. D. Panov et al., Energy spectra of abundant nuclei of primary cosmic rays from the data of ATIC-2 experiment: Final results, Bull. Russian Acad. of Science, Phys. 73 (2009) 564-567.

[15] H. S. Ahn et al., Discrepant Hardening Observed in Cosmic-ray Elemental Spectra, ApJ 714 (2010) L89-L93, [arXiv:1004.1123].

[16] M. Ackermann et al., Inferred Cosmic-Ray Spectrum from Fermi Large Area Telescope $\gamma$-Ray Observations of Earth's Limb, PRL 112 (2014) 151103.

[17] M. Aguilar and et al., Precision Measurement of the Proton Flux in Primary Cosmic Rays from Rigidity 1 GV to 1.8 TV with the Alpha Magnetic Spectrometer on the International Space Station, PRL 114 (2015) 171103.

[18] F. Donato, D. Maurin, P. Brun, T. Delahaye, and P. Salati, Constraints on WIMP Dark Matter from the High Energy PAMELA p $p^{-/ p}$ Data, PRL 102 (2009) 071301, [arXiv: 0810 . 5292].

[19] M. Cirelli, M. Kadastik, M. Raidal, and A. Strumia, Model-independent implications of the $e, p^{-}$ cosmic ray spectra on properties of Dark Matter, Nuclear Physics B 813 (2009) 1-21.

[20] T. A. Porter, R. P. Johnson, and P. W. Graham, Dark Matter Searches with Astroparticle Data, ARAA 49 (2011) 155-194, [arXiv:1104.2836].

[21] J. Arons, Particle acceleration by pulsars, in Origin of Cosmic Rays (G. Setti, G. Spada, and A. W. Wolfendale, eds.), vol. 94 of IAU Symposium, pp. 175-204, 1981.

[22] A. K. Harding and R. Ramaty, The Pulsar Contribution to Galactic Cosmic Ray Positrons, ICRC 2 (1987) 92. 
[23] A. Boulares, The nature of the cosmic-ray electron spectrum, and supernova remnant contributions, ApJ 342 (1989) 807-813.

[24] D. Hooper, P. Blasi, and P. Dario Serpico, Pulsars as the sources of high energy cosmic ray positrons, JCAP 1 (2009) 25, [arXiv:0810.1527].

[25] M. Di Mauro, F. Donato, N. Fornengo, R. Lineros, and A. Vittino, Interpretation of AMS-02 electrons and positrons data, JCAP 4 (2014) 6, [arXiv: 1402 .0321].

[26] E. G. Berezhko, L. T. Ksenofontov, V. S. Ptuskin, V. N. Zirakashvili, and H. J. Völk, Cosmic ray production in supernova remnants including reacceleration: The secondary to primary ratio, A\&A 410 (2003) 189-198, [astro-ph/0308199].

[27] P. Blasi, Origin of the Positron Excess in Cosmic Rays, PRL 103 (2009) 051104.

[28] P. Blasi and P. D. Serpico, High-Energy Antiprotons from Old Supernova Remnants, PRL 103 (2009) 081103, [arXiv:0904.0871].

[29] I. Cholis and D. Hooper, Constraining the origin of the rising cosmic ray positron fraction with the boron-to-carbon ratio, PRD 89 (2014) 043013, [arXiv: 1312 . 2952].

[30] P. Mertsch and S. Sarkar, AMS-02 data confront acceleration of cosmic ray secondaries in nearby sources, PRD 90 (2014) 061301, [arXiv: 1402 .0855].

[31] M. Kachelrieß, S. Ostapchenko, and R. Tomàs, Antimatter Production in Supernova Remnants, ApJ 733 (2011) 119, [arXiv:1103.5765].

[32] M. Kachelrieß and S. Ostapchenko, B/C ratio and the PAMELA positron excess, PRD 87 (2013) 047301, [arXiv:1211.1033].

[33] M. Kadastik, M. Raidal, and A. Strumia, Enhanced anti-deuteron Dark Matter signal and the implications of PAMELA, Physics Letters B 683 (2010) 248-254, [arXiv: 0908 . 1578 ].

[34] L. A. Dal and M. Kachelrieß, Antideuterons from dark matter annihilations and hadronization model dependence, PRD 86 (2012) 103536, [arXiv: 1207 .4560].

[35] M. Kachelriess, I. V. Moskalenko, and S. S. Ostapchenko, New Calculation of Antiproton Production by Cosmic Ray Protons and Nuclei, ApJ 803 (2015) 54, [arXiv: 1502.0415$].$

[36] L. C. Tan and L. K. Ng, Parametrisation of hadron inclusive cross sections in p-p collisions extended to very low energies, Journal of Physics G Nuclear Physics 9 (1983) 1289-1308.

[37] R. P. Duperray, C.-Y. Huang, K. V. Protasov, and M. Buénerd, Parametrization of the antiproton inclusive production cross section on nuclei, PRD 68 (2003) 094017, [astro-ph/ 0305274 ].

[38] M. Kachelriess, I. V. Moskalenko, and S. S. Ostapchenko, Nuclear Enhancement of the Photon Yield in Cosmic Ray Interactions, ApJ 789 (2014) 136, [arXiv: 1406.0035$].$

[39] M. Honda, T. Kajita, K. Kasahara, and S. Midorikawa, New calculation of the atmospheric neutrino flux in a three-dimensional scheme, PRD 70 (2004) 043008, [a stro-ph/ 0404457 ].

[40] I. V. Moskalenko, A. W. Strong, and O. Reimer, Diffuse galactic gamma rays, cosmic-ray nucleons and antiprotons, A\&A 338 (1998) L75-L78, [astro-ph/ 9808084 ].

[41] A. W. Strong, I. V. Moskalenko, and V. S. Ptuskin, Cosmic-Ray Propagation and Interactions in the Galaxy, Ann. Rev. Nucl. Part. Sci. 57 (2007) 285-327, [astro-ph/0701517].

[42] A. E. Vladimirov and et al., GALPROP WebRun: An internet-based service for calculating galactic cosmic ray propagation and associated photon emissions, Computer Phys. Comm. 182 (2011) 1156-1161, [arXiv:1008.3642]. 